\title{
Wavelet-based 3-D Multifractal Spectrum with Applications in Breast MRI Images
}

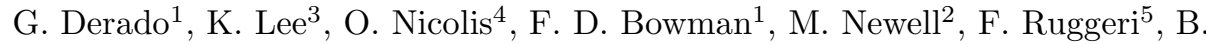 \\ Vidakovic $^{3}$ \\ 1 Emory University, Atlanta, GA \\ 2 Winship Cancer Institute, Atlanta, GA, \\ 3 Georgia Institute of Technology and Emory University, Atlanta, GA, \\ 4 University of Bergamo, Italy \\ 5 CNR Milano, Italy
}

\begin{abstract}
Breast cancer is the second leading cause of death in women in the United States. Breast Magnetic Resonance Imaging (BMRI) is an emerging tool in breast cancer diagnostics and research, and it is becoming routine in clinical practice. Recently, the American Cancer Society (ACS) recommended that women at very high risk of developing breast cancer have annual BMRI exams, in addition to annual mammograms, to increase the likelihood of early detection. (Saslow et al. [20]). Many medical images demonstrate a certain degree of self-similarity over a range of scales. The multifractal spectrum (MFS) summarizes possibly variable degrees of scaling in one dimensional signals and has been widely used in fractal analysis. In this work, we develop a generalization of MFS to three dimensions and use dynamics of the scaling as discriminatory descriptors for the classification of BMRI images to benign and malignant. Methodology we propose was tested using breast MRI images for four anonymous subjects (two cancer, and two cancer-free cases). The dataset consists of BMRI scans obtained on a 1.5T GE Signa MR (with VIBRANT) scanner at Emory University. We demonstrate that meaningful descriptors show potential for classifying inference.
\end{abstract}

\section{Introduction}

In the United States, breast cancer is the second leading cause of death in women (after lung cancer), and is the most common cancer among women. One out of eight women will develop breast cancer in their lifetime. The American Cancer Society (ACS) estimated that about 40,460 women would die from the disease in 2007 (Jemal et al. [10]). Studies have indicated that early detection and treatment improve the chances of survival for breast cancer patients (Curpen et al. [6], Smart et al. [21]). Breast imaging plays a vital role in screening for and diagnosis of breast cancer and in monitoring the impact of treatment. In this study, we target the development of analytical techniques to improve diagnostic capabilities of BMRI.

While mammography and breast ultrasound are considered "gold standard" for breast cancer screening, an increasing body of research has shown BMRI to 
be an effective diagnostic and interventional tool. BMRI has been approved by the U.S. Food and Drug Administration since 1991 for use as a supplemental tool to mammography for breast cancer diagnostics. It is also useful in breast cancer staging, in treatment and preoperative planning, and for patient followup after breast cancer treatment. Since 1999, there has been a $40 \%$ per year increase in the number of BMRI examinations in the United States. Recently, ACS recommended that women at very high risk of developing breast cancer have annual BMRI exams, in addition to annual mammograms, to increase the likelihood of early detection (Saslow et al. (2007) [20]).

Based on the principles of nuclear magnetic resonance (NMR), a technique that is highly sensitive to physical, chemical and biological characteristics of tissues and fluids, BMRI enables a 3-D examination of breast tissue and provides a noninvasive assessment of the microcirculatory characteristics of tissues, in addition to traditional anatomical information. The 3-D anatomical structure, however, is insufficient for distinguishing between benign and malignant tissues, and functional imaging is typically incorporated. In this setting, functional imaging utilizes contrast agents for MRI, which enables the visualization of functional changes when serial MRI scans are acquired. The typical contrast agent for BMRI is Gadolinium (GAD) diethylenetriamine penta-acetic acid (DTPA).

Evaluating BMRI accurately and efficiently is essential, but it is very challenging in practice. BMRI produces massive 4-dimensional (three spatial dimensions plus a time dimension) data, posing challenges for analysis and detection. At present, BMRI cannot always distinguish between cancerous and non-cancerous functional dynamics, prompting the investigation into improved methods.

Wavelet techniques have become indispensable for image processing, in particular when dealing with medical images. Mallat's multiresolution analysis (see Vidakovic [22]) decomposes an image into a set of approximation coefficients (low frequency components) and the scale dependent hierarchy of detail coefficients (high frequency components). A standard tensor product orthogonal wavelet transformation of an image results in three sets of generated detail coefficients: diagonal, horizontal and vertical. Numerous references can be found in the literature in which wavelets are applied to mammogram images. For example, in Yoshida et al. [23], a wavelet transform was applied to detect clustered microcalcifications. In Zheng et al. [24] and Derado et al. [5], a wavelet-based image-enhancement method is employed to enhance microcalcification clusters for improved detection. Recently there has been an increase in the use of waveletbased methodology in the analysis of BMRI data. Alterson and Plewes (2003)[1] used a multiresolution non-orthogonal wavelet representation as a measure of similarity to detect natural biological symmetries in breast MRI scans. Mainardi et al. (2007) [13] present a nonrigid registration algorithm of dynamic MR breast images based on a multiresolution motion estimation of the breast using complex discrete wavelet transform. To the best of our knowledge, however, approaches using scaling methodology in BMRI data can not be found in the published literature. 
Fractality is a concept pervasive in medical research. Many medical signals and images demonstrate a certain degree of self-similarity over a range of scales, lending to the development of algorithms based on fractal analysis of those objects (see Chen et al. [4] and Kuklinski [12]). For example, fractality was used to detect breast cancer in Priebe et al. [16], Kestener et al. [11], and Bocchi et al. [2]. Chen et al. [4] developd a pattern recognition technique based on features derived from the fractal description of mammograms. Kuklinski [12] used a wavelet transform modulus maxima method generalized to the two dimensional case. They combined this approach with a multifractal analysis, enabling the detection of tumors as well as microcalcifications. Kestener et al. [11] used long range correlations and wavelet-based multifractality for tissue classification in digitized mammograms to support clinical diagnosis. In Moloney et al. [14], the MFS is used to analyze the pupillary behavior of older adults and to discriminate between patients with various ocular acuity.

Processes with fractal characteristics that exhibit rich scaling behavior are often referred to as multifractals. The fractional Brownian motion, a theoretical model for mono-fractality, is a non-stationary process whose sample paths exhibit a homogeneous degree of regularity. For many applications, this homogeneous regularity may be too restrictive. In particular, one may want models that account for differing degrees of regularity. Multifractal analysis is concerned with describing the local singular behavior of functions in a geometrical and statistical fashion. It was first introduced in the context of turbulence and applied in many other contexts such as Diffusion Limited Aggregation (DLA) patterns research, earth quake distribution analysis, signal processing and internet data traffic modeling. For an introduction to multifractals, see Riedi [18]. Multifractal models exhibit patterns of locally varying scaling behavior similar to that encountered in medical and biological data (among others). They usually exhibit a prevalent scaling behavior, but a multitude of other scalings may also be present although occurring much less frequently. Since multifractal models are in general non-stationary, standard tools in time series analysis such as the Fourier transform are not appropriate because the Fourier transform is not localized in time. Evaluating the varying local properties of multifractal processes requires analytical methods that are able to localize information in time and frequency. Given that wavelets are local in both frequency/scale (via dilations) and in time (via translations), the wavelet defined multiscale analysis is convenient in assessing multitude scalings intrinsic for BMRI scans. For a detailed study of multifractals, we refer the reader to Riedi [17] and Morales [15].

The multifractal spectrum (MFS) summarizes possibly variable degrees of scaling in signals. In the case of fractals, scaling refers to the propagation of energy when the signals or images are inspected at various resolutions. The dynamics of the scaling can be used as discriminatory descriptors; thus, multifractality provides an additional window through which to look at the data and renders standard statistical approaches insufficient.

In this work, we generalize the concept of multifractal spectrum as it was defined in Gonçalves et al. [9] to the three dimensional case and use some of 
its low-dimensional descriptors to classify BMRI scans as either benign or malignant. Although the number of subjects analyzed is small (two cases and two controls), our findings are consistent with empirical evidence that healthy responses are characterized by irregularity and that increased regularity may suggest pathologies.

The paper is organized as follows. Section 2 gives a description of the data to which we apply our proposed method. In Section 3, we provide a brief review of the theoretical background of wavelets In addition, the three dimensional multifractal spectrum is defined and some of its properties are illustrated on the example of 3-D fractional Brownian motion MFS. Section 4 deals with the application of our proposed methodology to cancer detection via the classification of BMRI. In Section 5 we provide conclusions and delineate some possible directions for future research.

\section{Description of the data}

The data consist of serial BMRI scans from each of four women: 2 cancer and 2 cancer-free cases. The scan series includes one pre-contrast image and four post-contrast images acquired at 1, 3, 5, and 7 minutes after the contrast is administered. The discriminatory pattern of contrast enhancement, characterized by rapid accumulation in the malignant mass and rapid wash out, occurs in the first few minutes following injection. By 7 minutes or later, the contrast uptake in most breast tissue is enhancing. Each 3-D scan contains 104 such sagittal slices comprised of an array of $256 \times 256$ pixels and slice thickness of $3 \mathrm{~mm}$. The scans were obtained on a $1.5 \mathrm{~T}$ GE Signa MR (with VIBRANT) scanner at Emory University.

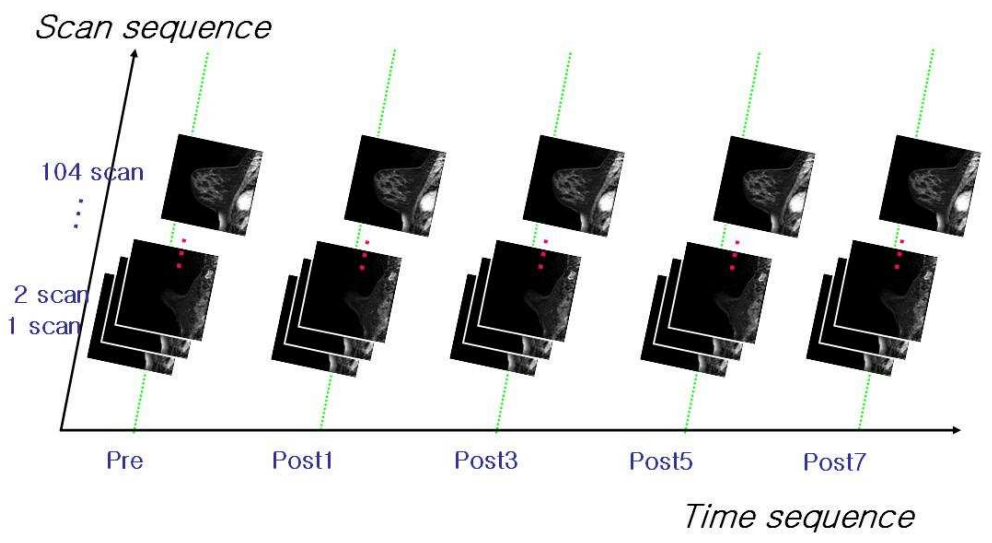

Fig. 1. Illustration of the data structure and acquisition 


\section{Methodology}

In this work we present a conceptual description of MFS in three dimension and demonstrate its utility in the classification of BMRI images. Our approach consists of two main steps. First, we estimate the multifractal spectra and extract a number of low dimensional summaries (such as slopes, tangents, broadness and spectral mode). Then, we use these summaries as discriminatory measures for BRMI images.

\subsection{Background on wavelets}

The 3-D wavelet basis functions are constructed via translations and dilations of a tensor product of univariate wavelets and scaling functions. For technical reasons, we consider $L^{1}$-normalization of wavelets instead of standard $L^{2}$ normalization, of which expression for $\psi_{j, \mathbf{k}}, \phi_{j, \mathbf{k}}$ is,

$$
\begin{aligned}
& \phi_{j, \mathbf{k}}(\mathbf{x})=2^{3 j} \phi\left(2^{j} x_{1}-k_{1}, 2^{j} x_{2}-k_{2}, 2^{j} x_{3}-k_{3}\right) \\
& \psi_{j, \mathbf{k}}^{i}(\mathbf{x})=2^{3 j} \psi^{i}\left(2^{j} x_{1}-k_{1}, 2^{j} x_{2}-k_{2}, 2^{j} x_{3}-k_{3}\right)
\end{aligned}
$$

where $i=h, l, v, h l, h v, l v, h l v$ denote the different directions on a cube (see Fig. 2 , left $), \mathbf{x}=\left(x_{1}, x_{2}, x_{3}\right) \in \mathbf{R}^{3}$, and $\mathbf{k}=\left(k_{1}, k_{2}, k_{3}\right) \in \mathbf{Z}^{3}$. Then, any function $f \in \mathbf{L}_{2}\left(\mathbf{R}^{3}\right)$ can be represented as

$$
f(\mathbf{x})=\sum_{\mathbf{k}} c_{j_{0} \mathbf{k}} \phi_{j_{0}, \mathbf{k}}(\mathbf{x})+\sum_{j \geq j_{0}} \sum_{\mathbf{k}} \sum_{i} d_{j, \mathbf{k}}^{i} \psi_{j, \mathbf{k}}^{i}(\mathbf{x})
$$

where the wavelet coefficients are given by

$$
d_{j, \mathbf{k}}^{i}=2^{3 j} \int f(\mathbf{x}) \psi^{i}\left(2^{j} \mathbf{x}-\mathbf{k}\right) \mathrm{d} \mathbf{x}
$$

The 3-D multifractal wavelet spectra will be defined using the wavelet coefficients $d_{j, \mathbf{k}}^{i}$, along the scale index $j$. We assume that the mother wavelet $\psi$ has $\mathcal{R}$ vanishing moments, that is, $\int x^{r} \psi(x) d x=0, r=0, \ldots, \mathcal{R}$, because the decorrelation property of wavelet coefficients depends upon this assumption.

Although the wavelet analysis of $n$-dimensional structures is conceptually straightforward, it is not routinely implemented in standard wavelet software and for this project we developed and implemented the three dimensional transformation in MATLAB's freely available package Wavelab [3].

\subsection{3-D Multifractal spectrum}

In Gonçalves et al. [9], it is shown how the oscillatory or scaling behavior of a process carries over into the local scaling properties of its wavelet coefficients $d_{j, \mathbf{k}}^{i}$ in (2), under assumption that the wavelet is more regular than the process. The following local singularity strength measure in 3-D can be defined using wavelets

$$
\alpha^{i}(\mathbf{t}):=\lim _{\mathbf{k} 2^{-\mathbf{j}} \rightarrow \mathbf{t}}-\frac{1}{j} \log _{2}\left|d_{j, \mathbf{k}}^{i}\right|
$$



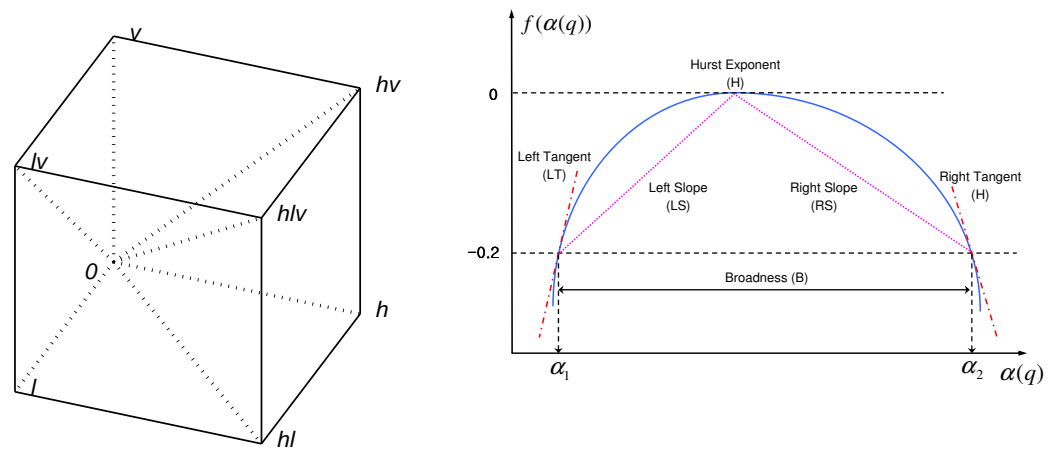

Fig. 2. Seven directions on a cube corresponding to detail level hierarchies in a $3-\mathrm{D}$ wavelet transforms (left); Summary measured (descriptors) from a schematic multifractal spectrum (right).

where $\mathbf{k} 2^{-j} \rightarrow \mathbf{t}$ means that $\mathbf{t}=\left(t_{1}, t_{2}, t_{3}\right) \in\left[2^{-j} k_{1}, 2^{-j}\left(k_{1}+1\right)\right) \times\left[2^{-j} k_{2}, 2^{-j}\left(k_{2}+\right.\right.$ $1)) \times\left[2^{-j} k_{3}, 2^{-j}\left(k_{3}+1\right)\right)$ for $\mathbf{k}=\left(k_{1}, k_{2}, k_{3}\right)$ and $j \rightarrow \infty$. Smaller $\alpha(\mathbf{t})$ corresponds to larger oscillations in $X$ and thus to more singularity at time t. The index $i$ in (3) corresponds to one of seven directions in detail spaces of 3-D wavelet transform, horizontal (h), vertical (v) or diagonal (d) up to horizontal, vertical, diagonal (hvd). Typically, a process will possess many different singularity strengths. The frequency (in t) of occurrence of a given singularity strength $\alpha$ is measured by the $3 D$ multifractal spectrum, defined for each direction $i$ as

$$
\begin{aligned}
f^{i}(\alpha) & :=\lim _{\epsilon \rightarrow 0} \lim _{j \rightarrow \infty} \frac{1}{j} \log _{2} M_{j}^{i} \\
M_{j}^{i} & :=2^{-j} \#\left\{\mathbf{k}: 2^{-j(\alpha+\epsilon)} \leq\left|d_{j, \mathbf{k}}^{i}\right| \leq 2^{-j(\alpha+\epsilon)}\right\} .
\end{aligned}
$$

For $\mathbf{k} \in\left\{0, \ldots, 2^{j}-1\right\} \times\left\{0, \ldots, 2^{j}-1\right\}, f^{i}$ takes values between -1 and 0 . Smaller $f^{i}(\alpha)$ means that "fewer" points $\mathbf{t}$ behave with strength $\alpha(\mathbf{t}) \simeq \alpha$.

The 3-D multifractal spectrum $f^{i}$ defined in (4) is very hard to calculate. A simpler approach makes use of the theory of large deviations (see Ellis, [8]), where $f^{i}$ would be interpreted as the rate function of a Large Deviation Principle: $f^{i}$ measures how frequently (in $\mathbf{k}$ ) the observed $(-1 / j) \log _{2}\left|d_{j, \mathbf{k}}^{i}\right|$ deviate from the "expected value" $\alpha_{0}$ in scale $j$. In our 3 -D context, it corresponds to studying the scaling behavior of the moments of the wavelets coefficients (2). For every direction $i$, the partition function is defined,

$$
T^{i}(q):=\lim _{j \rightarrow \infty}(-1 / j) \log _{2} \mathbf{E}\left|d_{j, \mathbf{k}}^{i}\right|^{q}
$$

It describes limiting behavior of qth moment of a typical wavelet coefficient $d_{j, \mathbf{k}}^{i}$ from the level $j$ and direction $i$. The multifractal formalism posits that the multifractal spectrum can be calculated by taking the Legendre transform of the 
corresponding log moment generating function (Riedi et al. [19])

$$
f^{i}(\alpha)=f_{L}^{i}(\alpha):=\inf _{q}\left[q \alpha-T^{i}(q)\right] .
$$

It can be shown that $f_{L}^{i}(\alpha)=q \alpha-T^{i}(q)$ at $\alpha^{i}=T^{\prime} i(q)$ provided $T^{\prime \prime} i(q)<0$.

\subsection{Wavelet-based estimator}

We discuss in this section wavelet-based estimation of the 3-D multifractal spectrum (4). Given a realization of the 3-D fBm of size $2^{J} \times 2^{J} \times 2^{J}$, and using the stationarity of the wavelets coefficients $\left\{d_{j,\left(k_{1}, k_{2}, k_{3}\right)}^{i}, i=h, l, v, h l, h v, l v, h l v ; j=\right.$ $\left.J_{0}, \ldots, J-1, k_{1}, k_{2}, k_{3}=0, \ldots, 2^{j}-1\right\}$, the sample counterpart of $\mathbf{E}\left|d_{j, \mathbf{k}}^{i}\right|^{q}$ is

$$
\widehat{S}_{j}^{i}(q):=\frac{1}{2^{3 j}}\left(\sum_{k_{1}=0}^{2^{j}-1} \sum_{k_{2}=0}^{2^{j}-1} \sum_{k_{3}=0}^{2^{j}-1}\left|d_{j,\left(k_{1}, k_{2}, k_{3}\right)}^{i}\right|^{q}\right)
$$

for $q>-1$. The partition function can then be estimated as the power-law exponent of the variation of $\hat{S}_{j}^{i}(q)$ versus scale $2^{-j}$. By linear regression of $\log _{2} \hat{S}_{j}^{i}(q)$ on $j$ between scales $j_{1}$ and $j_{2}$ we get

$$
\widehat{T}^{i}(q):=\sum_{j=j_{1}}^{j_{2}} a_{j} \log _{2} \widehat{S}_{j}^{i}(q),
$$

where the regression weights $a_{j}$ must verify the two conditions $\sum_{j} a_{j}=0$ and $\sum_{j} j a_{j}=1$ (Delbeke and Abry [7]). Thus, we can estimate $f^{i}(\alpha)$ though a local slope of $\widehat{T}^{i}(q)$ at values

$$
\widehat{\alpha}^{i}\left(q_{l}\right)=\left[\widehat{T}^{i}\left(q_{l+1}\right)-\widehat{T}^{i}\left(q_{l}\right)\right] / q_{0}, \quad q_{l}=l q_{0}
$$

as

$$
\widehat{f}^{i}\left(\alpha^{i}\left(q_{l}\right)\right)=q_{l} \alpha^{i}\left(q_{l}\right)-\widehat{T}^{i}\left(q_{l}\right) .
$$

Multifractal spectra can be found even for monofractal processes, the spectra generated from monofractal processes are ramp-like with a dominant (modal) irregularity corresponding to the theoretical Hurst exponent (see Riedi [17]).

Rather than operating with multifractal spectra as functions (densities), we summarize them by a small number of meaningful descriptors. Each multifractal spectrum (in each direction) can be approximately described by 3 canonical descriptors without loss of the discriminant information, which are (1) Spectral Mode (Hurst exponent, $H)$, (2) left slope $(L S)$ or left tangent $(L T)$ and (3) width spread (Broadness, $B)$ or right slope $(L S)$ or right tangent $(R T)$. A typical multifractal spectrum can be quantitatively described as shown in Figure 3. Understanding the $H$ and $L S$ (or $L T$ ) is straightforward. $H$ represents the apex of the spectrum or the Hurst exponent, and $L S$ (or $L T$ ) represents the slope of the distribution produced by the collection of Hurst exponents with smaller 
values of the mode $(H)$. However, broadness $(B)$ is a more intricate descriptor of the multifractal spectrum. Broadness $(B)$ is believed to be more meaningful than right slope $(R S)$ or right tangent $(R T)$ because it is a compound measure representing the overall nature of the multifractal spectra, taking into account the overall variability among the Hurst exponents. In addition, broadness $(B)$ partially accounts for right slope $(R S)$ or right tangent $(R T)$ in calculation, as the resultant value of $B$ is based on the relative values of $R S$ and $L S$. Both slopes (or both tangents) can be obtained easily using the interpolation technique, while it is not straightforward to define the broadness $(B)$ automatically. The location of the start and end points of the width spread has been set to the roots $\alpha_{1}$ and $\alpha_{2}$ which satisfy the equation $f(\alpha)+0.2=0$ as in (Shi et al. 2006). Figure 3 depicts the MFS of a simulated 3-D fBm with $H=0.3$ and $H=0.7$. Notice how the maximum of every $f^{i}(\alpha)$ is attained close to $\alpha=0.3$ and $\alpha=0.7$, and deviations from the exact values can be attributed to discretization or small number of dyadic levels.
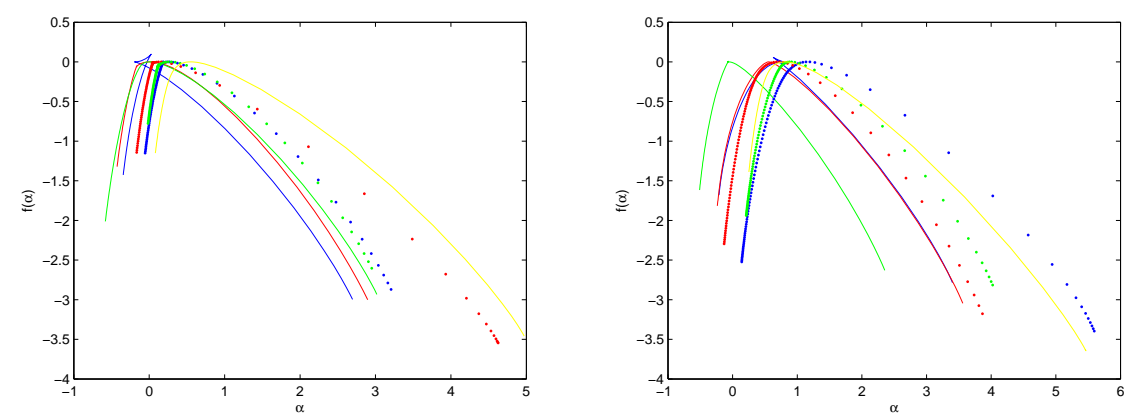

Fig. 3. 3D MFS associated to a $3 \mathrm{D} \mathrm{fBm}$ with $H=0.3$ (left) and $H=0.7$ (right), respectively.

\section{Application in analysis of BMRI images}

In this section, we provide an application of the previously defined 3-D waveletbased multifractal spectrum to the classification of BMRI images. We classify images as benign or malignant, by analyzing the fractal properties of the background of the image. Each image was divided in non-overlapping subimages, each of size $256 \times 256 \times 256$. Each 3 -D image contains 104 slices of $256 \times 256$ scans that are boundary mirror extended to obtain "wavelet friendly" dimension of 256 .

Figure 4 displays $256 \times 256$ BMRI slices (cross-sections) from a cancer case and from a control (non-cancerous) subject. 

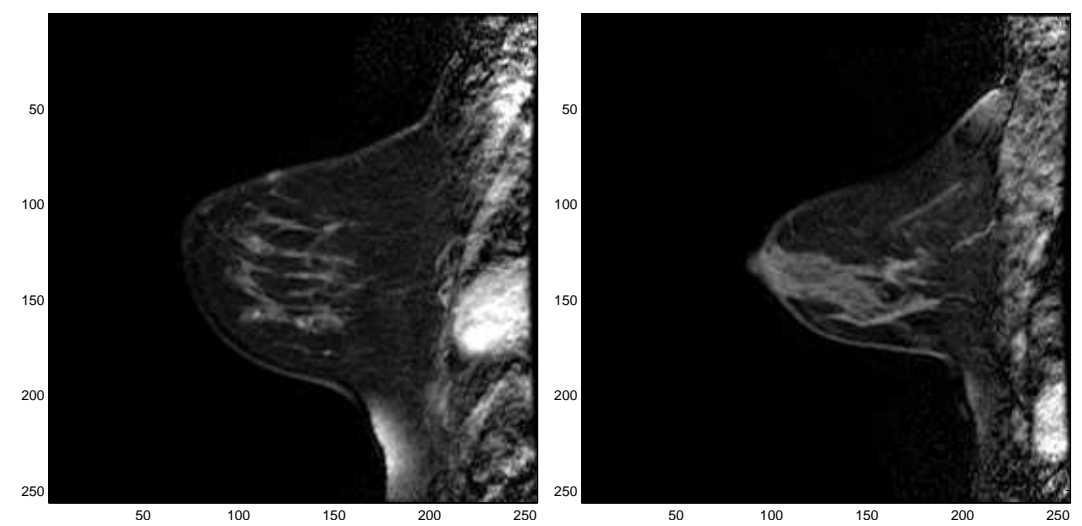

Fig. 4. Examples of case (left) and control (right) BMRI scans, one sagittal slice is shown.

Figure 5 shows particular multifractal descriptors (see also Fig. 2 and its caption) in selected directions for the BMRI data containing two cases and two controls. Fig. 5 (a) displays two selected descriptors, namely $H$ and $L S$, since they are easily interpretable and appear to distinguish features of cases and controls reasonably well. The descriptor $H$ measures the global irregularity of a scan, while $L S$ describes the deviation from mono-fractality. Images with higher $L S$ values exhibit greater mono-fractality, and a pure monofractal theoretically has an infinite $L S$. The direction $\mathrm{hlv}$, corresponding to the main diagonal, is selected since the hierarchy of wavelet $\mathrm{hlv}$-subspaces contains genuine details. That is, coefficients are obtained by applying high-pass filters in all 3 dimensions, while any other hierarchy utilizes at least one low-pass filter. It is evident that the controls are placed in the region for which $H+L S$ is small - indicating that both irregularity and multifractality for controls are high. This is consistent with a popular belief that increased regularity and monofractality are signs of pathology for many high frequency biometric responses (electrocardiogram (ECG), ocular responses, etc).

Fig. 5 (c) shows the longitudinal behavior of the broadness descriptor $B$ for the cases and controls along the direction $\mathrm{hv}$. The $\mathrm{hv}$ direction combines $\mathrm{h}$ and $\mathrm{v}$, which are sampled along directions within slices and between the slices, respectively. The descriptor $B$ is conceptually linked to $H, R S$ and $L S$. Namely, spectra with low $H$ and small $L S$ tend to have large $B$. These typical associations are consistent with our findings depicted in Fig. 5 (a). Even with a small sample size, Figure 5 illustrates the discriminatory power of the multifractal descriptors in BMRI applications. In particular, Fig. 5 (b) shows a non-linear decision boundary generated by a support vector machine (SVM) classifier with radial basis kernel of width parameter 0.1. In our application, the SVM classifier achieved $95 \%$ accuracy. 


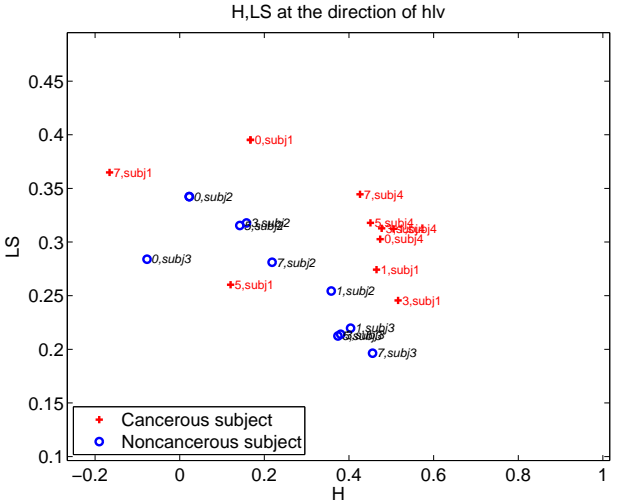

(a) Realization of indices $(H, L S)$; the symbols denote a time index, a subject numer, and cancer status.

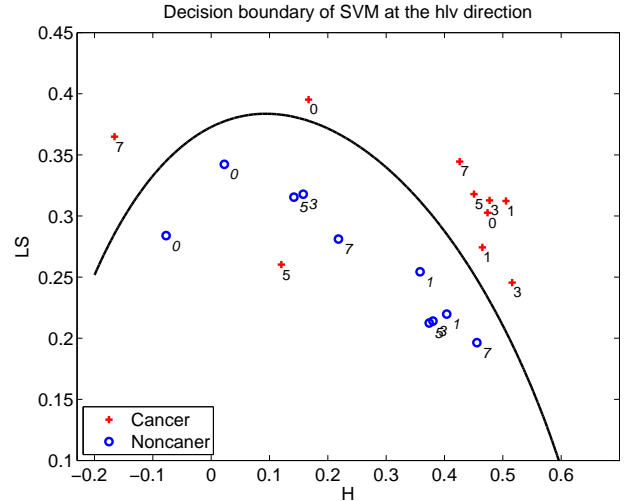

(b) A decision boundary of SVM (radial basis of width 0.1 ); the symbols denote a time index and cancer status.

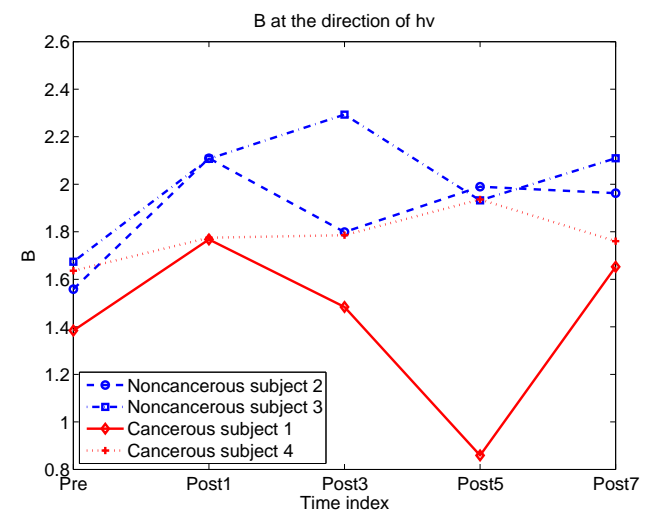

(c) Longitudinal behavior of the descriptor $B$

Fig. 5. Multifractal descriptors and an associated classifier in selected directions for two cases and two controls. Images (a) and (b) use the main hlv-direction, while the third image (c) uses the hv-direction.

\section{Conclusions}

In this work, we have shown that the extended three dimensional concept of wavelet-based multifractal spectrum can be utilized in classification of BMRI. This tool, which describes various degrees of irregularity in the measured objects, has been widely utilized in several fields (e.g. physics, meteorology, and medicine), where assessing self-similarity and fractality is critical. Our methodology has provided promising results that are consistent with past research. For example, we observed in our data that normal breast tissue tends to be more irregular (with a smaller Hurst exponent) than tumor affected tissue. 
The findings in our study are based upon a small data set, for which the applicability of formal classification algorithms is limited. In future research, involving more data, we plan to build and apply a weak classifier based on scaling of BMRI background, which is a novel concept in cancer screening. We applied the flexible SVM classifier that allows for non-linear classification boundaries, and we will consider other state-of-the art methods in future research. Classification will become more statistically reliable with a large data set that we are in the process of obtaining.

Extremely high classification precision will be challenging to attain with a single classifier, given the high degree of noise in MRI measurements and numerical instability of our algorithms due to limited spatial resolution in the images. However, even moderately accurate classifiers may contribute substantially to breast cancer screening, and these so-called weak classifiers in our context utilize information (BMRI background) that is currently ignored and may combine with other weak classifiers (via boosting) to produce clinically useful tools.

\section{References}

1. Alterson, R. and Plewes, D. B. (2003). Bilateral symmetry analysis of breast MRI, Phys. Med. Biol., 48, 3431-3443.

2. Bocchi, L., Coppini, G., Nori, J. and Valli, G. (2004). Detection and clustered microcalcifications in mammograms using fractals models and neural networks. Medical Engineering \& Physics, 26, 303-312.

3. Buckheit, J. and Donoho, D. (1995). Wavelab and reproducible research. Technical report, Stanford University.

4. Chen, CC., Daponte, JS. and Fox, MD. (1989). Fractal features analysis and classification in medical imaging. IEEE Transactions on Medical Imaging,8, 133-142.

5. Derado, G., Bowman, F.D., Patel, R., Newell, M., and Vidakovic, B.: "Wavelet Image Interpolation (WII): A Wavelet-based Approach to Enhancement of Digital Mammography Images", Springer Verlag Lecture Notes in Bioinformatics ISBRA 2007, LNBI 4463, pp. 203-214, 2007 (Eds. I. Mandoiu and A. Zelikovsky).

6. Curpen, B. N., Sickles, E. A., Sollitto R. A. (1995). The comparative value of mammographic screening for women 40-49 years old versus women 50-59 years old. AJR, 164, 1099-1103.

7. Delbeke, L. and Abry, P. (1998). Wavelet-based estimators for the self-similar parameter of fractional Brownian motion. Submitted to Appl. Comp. Harm. Anal.

8. Ellis, R. (1984). Large deviations for a general class of random vectors. Ann. Prob, $12,1-12$.

9. Gonçalves P., Riedi, R. and Baraniuk, R. (1998). Simple statistical analysis of waveletbased multifractal spectrum estimation. In Proceedings 32nd Asilomar Conference on Signals, Systems and Computers, Pacific Grove, CA.

10. Jemal, A., Siegel, R., Ward, E., Murray, T., Xu, J., Thun, M. J. (2007). Cancer Statistics, CA Cancer J Clin, 2007; 57:4366.

11. Kestener, P., Lina, J.M., Saint-Jean, P. and Arneodo, A. (2001). Wavelet-based multifractal formalism to assist in diagnosis in digitized mammograms. Image Anal Stereol, 20, 169-174.

12. Kuklinski, W.S. (1994). Utilization of fractal image models in medical image processing. Fractals, 2, 363-369. 
13. Mainardi, L., Passera, K. M., Lucesoli, A., Vergnaghi, D., Trecate, G., Setti, E., Musumeci, R. and Cerutti, S., A Nonrigid Registration of MR Breast Images Using Complex-valued Wavelet Transform, Journal of Digital Imaging, Springer New York, published online 28 February 2007.

14. Moloney, K.P, Jacko, J.A, Vidakovic, B., Sainfort, F, Leonard, V.K, and Shi, B. (2006). Leveraging data complexity: Pupillary behavior of older adults with visual impairment during HCI. ACM Trans. Comput.-Hum. Interact., 13:3, 376-402.

15. Morales, C. J. (2002). Wavelet-based multifractal spectra estimation: Statistical aspects and applications. Ph.D thesis. Boston University Graduate School of Arts and Sciences.

16. Priebe, C. E., Solka, J. L., Lorey, R. A., Rogers, G. W., Poston, W. L., Kallergi, M., Quian, W., Clarke, L. P. and Clark, R. A. (1994). The application of fractal analysis to mammographic tissue classification. Cancer letters, 77, 183-189.

17. Riedi, R. (2002). Multifractal Processes in Theory and Applications of Long-Range Dependence. Paul Doukhan, George Oppenheim, Murad S. Taqqu Editors. InPress, 625-716.

18. Riedi, R. H. (1999). An Introduction to multifractals, Tech Report, Dept Statistics Rice University.

19. Riedi, R., Crouse, M.S., Ribeiro, V., and Baraniuk, R.G. (1999). A multifractal wavelet model with applications to TCP network traffic. IEEE Trans. Info. Theory (special issue on multiscale statistical signal analysis and its applications).

20. Saslow, D., Boetes, C., Burke, W., Harms, S., Leach, M. O., Lehman, C. D., Morris, E., Pisano, E., Schnall, M., Sener, S., Smith, R. A., Warner, E., Yaffe, M., Andrews, K.S., Russell, C. A., MD for the American Cancer Society Breast Cancer Advisory Group (2007), American Cancer Society Guidelines for Breast Screening with MRI as an Adjunct to Mammography, CA Cancer J Clin ; 57:75-89.

21. Smart, C. R., Hendrick, R. E., Rutledge J. H., and Smith, R. A. (1995). Benefit of mammography screening in women ages 40 to 49 years: current evidence from randomized controlled trials. Cancer, 75, 1619-1626.

22. Vidakovic, B. (1999). Statistical Modeling by Wavelets. Wiley, NY, USA.

23. Yoshida, H., Doi, K., Nishikawa, R.M., L.Giger, M. and A.Schmidt, R. (1996). An improved computer-assisted diagnosis scheme using wavelet transform for detecting clustered microcalcifications in digital mammograms. Acad. Radiol., 3, 621-627.

24. Zheng, B., Qian, W. and Clarke, L.P. (1996). Digital mammography: mixed feature neural network with spectral entropy decision for detection of microcalcifications. Med. Img., 15, 589-597. 